\title{
Self-Assembled Surfactants on Patterned Surfaces: Confinement and Cooperative Effects on Aggregates Morphology
}

\author{
Manaswee Suttipong, ${ }^{\mathrm{a}}$ Brian P. Grady, ${ }^{\mathrm{b}}$ and Alberto Striolo ${ }^{\mathrm{a},}$, \\ ${ }^{a}$ Department of Chemical Engineering, University College London, London WC1E 6BT, UK.
}

${ }^{\mathrm{b}}$ School of Chemical, Biological and Materials Engineering, University of Oklahoma, Norman, Oklahoma 73069, USA.

* Author to whom correspondence should be addressed: $\underline{\text { a.striolo@ ucl.ac.uk }}$ 


\section{ABSTRACT}

The adsorption and self-assembly of surfactants are ubiquitous processes in several technological applications, including the manufacture of nano-structured materials using bottom-up strategies. Although much is known about the adsorption of surfactants on homogeneous flat surfaces from experiments, theory, and/or simulations, limited information is available, in quantifiable terms, regarding the adsorption of surfactants on surfaces with chemical and/or morphological heterogeneity. In an effort to fill this knowledge gap, we report here results obtained using equilibrium dissipative particle dynamics (DPD) simulations for the adsorption of model surfactants onto patterned flat surfaces (i.e., flat surfaces with chemical heterogeneity). The patterns consist of one or two stripes of variable width on which the surfactants could adsorb. The adsorbing stripes are surrounded by a surface that effectively repels the surfactants. This repelling surface, perhaps not realistic, allows us to quantify the effect of lateral confinement on the morphology of surfactant aggregates. When the stripe width is large (effectively providing a homogeneous flat surface), the surfactants yield a flat monolayer. Our simulations suggest that the flat monolayers become hemicylinders, hemi-spheres, and individual surfactants as the stripe width decreases, a consequence of lateral confinement. In some cases our simulations show evidence of cooperative effects when two adsorbing stripes are present on the surface. If the distance between the stripes and the widths of the stripes are both less than about one surfactant length, hemi-cylindrical shells and irregular structures are observed because of cooperativity; otherwise the results match those found for a single isolated stripe. Our predictions could be useful for the design of new nano-structured materials and coatings, for applications ranging from nano-fluidic devices to nano-reactors.

Keywords: Surfactant self-assembly; Nano-patterned surfaces; DPD simulations 


\section{Introduction}

The widespread interest in the self-assembly of surfactants on solid surfaces is motivated by many industrial processes. ${ }^{1-7}$ To describe surfactant self-assembly, the relationship between surfactant properties, surface characteristics, and adsorption thermodynamics must be understood. Many interesting experimental ${ }^{8-13}$ and computational ${ }^{14-22}$ results concerning surfactant adsorption on flat homogeneous surfaces have been reported in the literature. However, very little has been carefully quantified regarding surfactant adsorption on heterogeneous surfaces. Fundamental questions that might lead to important innovations include, but are not limited to: what is the effect of chemical surface heterogeneity on the amount of surfactants adsorbed? Are the morphologies of surfactant aggregates self-assembled on heterogeneous surfaces different compared to those obtained on flat homogeneous surfaces? And, ultimately, how can surfactant aggregates be manipulated by patterning a surface?

Surface heterogeneity can be caused by geometrical structures (e.g., surface roughness, shapes and sizes of surface features) and varying chemical composition (e.g., lattice defects, surface functional groups, and impurities). At the atomic scale geometrical heterogeneity must be associated with chemical heterogeneity, but chemical heterogeneity can in principle occur without geometrical heterogeneity (i.e., flat surfaces can be characterized by different chemical properties). Some experimental data suggest that surface heterogeneity can lead to rich surfactant behavior. For example, Foisner et al. found that self-assembled monolayers of octadecyltrichlorosilane (OTS) on modified silicon surfaces change to circular island structures when the surface is hydrophobized. ${ }^{23}$ Schniepp et al. showed that the orientation of sodium dodecylsulfate (SDS) surfactants on $\operatorname{Au}(111)$ surfaces strongly depends on surface roughness. ${ }^{24,}{ }^{25} \mathrm{Wu}$ et al. reported that surface roughness affects the amount of cetyltrimethylammonium bromide (CTAB) surfactant adsorbed on gold, as well as the visco-elastic properties of the self-assembled aggregates. ${ }^{26}$ 
From a theory and simulation point of view, a few authors addressed the adsorption of surfactants on heterogeneous surfaces. Using lattice Monte Carlo (MC) simulations, Reimer et al. ${ }^{27}$ investigated the adsorption of surfactants on surfaces in which a hydrophobic (hydrophilic) domain was surrounded by a hydrophilic (hydrophobic) surface, as well as on checkerboard hydrophobichydrophilic surfaces. They found that the dimension of the surface patterns influences the adsorption behavior of surfactants. Zhang et al. ${ }^{28}$ showed that the surfactant aggregates formed on heterogeneous (checkerboard and striped) surfaces are sufficiently dependent on the surface features that they could be used for surface-recognition purposes. Striolo ${ }^{29}$ reported that surfactant adsorption can be problematic when the size of the adsorbing square patch on a surface is less than a surfactant-dependent threshold. Tummala et $\mathrm{al}^{30}$ conducted molecular dynamics (MD) simulations to investigate the morphology of SDS surfactants on graphene nano-sheets, suggesting that the size of the graphene supports has a strong effect on the surfactants morphology, because of lateral-confinement effects. In our prior work we investigated the adsorption of several aqueous surfactants on various carbon nanotubes, and we found that the nanotube diameter strongly influences the morphology of the aggregates, via a sort of lateral-confinement effect due to the cylindrical shape of the nanotubes. ${ }^{31-34}$

We are interested here on the adsorption of surfactants on flat, chemically heterogeneous surfaces. Surfactant adsorption is allowed on stripes (considered hydrophobic) surrounded by a surface that is energetically repulsive enough to prohibit surfactant adsorption if a surface is uniform in that energy. As the width of the hydrophobic stripe decreases, the degree of lateral confinement increases. We report our simulation results for the morphology of the surfactant self-assembled aggregates. The simulations are conducted within the equilibrium dissipative particle dynamics (DPD) formalism. At the expense of representing the surfactants with atomistic precision, DPD allows us to investigate large systems for extended periods of time. For all simulations, a sufficient amount of surfactant is present to maintain concentrations above the critical micelle concentration 
in the bulk supernatant, which allows us to compare surfactant aggregates obtained at comparable thermodynamic conditions.

The remainder of the paper is structured as follows. In Section 2 we provide details regarding the simulation models and methods we used. In Section 3 we discuss our main results, which show evidence of strong effects due to lateral confinement, and also some evidence of cooperative behavior when the adsorbing stripes are not too wide and are close to each other. Lastly, in Section 4 we summarize the main conclusions. Additional details regarding the model and system composition of all simulated systems are included as supporting information.

\section{Simulation Models and Methods}

Dissipative particle dynamics (DPD) simulations were carried out in the NVT ensemble using the package LAMMPS, as released on 14May12. ${ }^{35}$ Full details for the DPD methodology are provided elsewhere. ${ }^{36-38}$

Simulations are conducted for aqueous surfactants near a flat solid surface. Each simulated system is comprised by five interaction beads, representing water, surfactant headgroup, surfactant tailgroup, hydrophobic surface sites, and surfactant-repelling surface sites.

A schematic representation of the coarse-grained model implemented here to represent water and surfactant molecules is provided in Figure 1a. Because we arbitrarily chose the degree of coarse graining $\mathrm{N}_{\mathrm{m}}=5$ (i.e., one DPD water bead represents five water molecules), the volume of one water bead is $\sim 150 \AA^{3}{ }^{39}$ We assigned the reduced density $\rho=5$ beads $/ \mathrm{r}_{\mathrm{c}}{ }^{3}$, defined as the number of beads in a cubic volume of radius $r_{c}$. Accordingly, $r_{c}=9.0856 \AA$. As customary, the volume of each bead $\left(150 \AA^{3}\right)$ in the simulation is maintained constant. Consequently, because the volume of one sodium dodecyl sulfate (SDS) molecule is $\sim 410 \AA^{3},{ }^{40}$ one surfactant can be approximated with 
three DPD beads: one to represent the headgroup, and two to represent the tailgroups. The three beads are connected via harmonic bonds to yield a single chain. Because the van der Waals end-toend length of one SDS molecule $\left(\mathrm{L}_{\mathrm{s}}\right)$ is of $20.8 \AA{ }^{41},{ }^{41}$ we assigned the equilibrium bond length $\mathrm{r}_{0}=$ 10.4 $\AA$. As suggested by Denham et al., ${ }^{42}$ we set the spring constant $\mathrm{k}_{\mathrm{S}}=100 \mathrm{k}_{\mathrm{B}} \mathrm{T} / \mathrm{r}_{\mathrm{c}}{ }^{2}$, which yields a $1.2 \%$ standard deviation for the bond length around $r_{0}$. Note that, because electrostatic interactions are not explicitly taken into consideration in our simulations, the model is not intended to reproduce all physical properties observed for ionic surfactants. In particular, in our approach we do not model counter-ions.

In each simulated system two solid surfaces are positioned at $\mathrm{Z}=0 \mathrm{r}_{\mathrm{c}}$ and at $\mathrm{Z}=42 \mathrm{r}_{\mathrm{c}}$. For clarity, in the remainder of the manuscript we refer to the $\mathrm{X}$ and $\mathrm{Y}$ directions as those parallel to the surface, while the $\mathrm{Z}$ direction is the one perpendicular to the surface. The solid surface at $\mathrm{Z}=42 \mathrm{r}_{\mathrm{c}}$ is repulsive to all surfactants. The one located at $\mathrm{Z}=0 \mathrm{r}_{\mathrm{c}}$ is the one on which we investigate surfactant adsorption. A schematic representation of the solid surfaces is provided in Figure 1b. The surface is built with 14400 beads treated as rigid bodies. The surface beads are organized in a square lattice with nearest-neighbor distance $\mathrm{P}=0.35 \mathrm{r}_{\mathrm{c}}$ in $\mathrm{X}$ and $\mathrm{Y}$ directions. Four planes of solid beads are stacked on top of each other with interlayer distance $Q=0.35 \mathrm{r}_{\mathrm{c}}$. Two consecutive planes are shifted with respect to each other by the shift distance $\mathrm{R}=0.175 \mathrm{r}_{\mathrm{c}}$ along both $\mathrm{X}$ and $\mathrm{Y}$ directions. Simulations were performed in a rectangular box of dimensions $21 \times 21 \times 42 \mathrm{r}_{\mathrm{c}}{ }^{3}$, with periodic boundary conditions applied along $\mathrm{X}$ and $\mathrm{Y}$ directions. We repeated selected simulations in boxes twice as large along $\mathrm{X}$ and $\mathrm{Y}$ directions, obtaining results consistent with those discussed in the text below.

In Figure 1c, we present the patterned surfaces, located at $\mathrm{Z}=0 \mathrm{r}_{\mathrm{c}}$, on which surfactants can adsorb. These heterogeneous surfaces are composed by repelling and hydrophobic (adsorbing) beads. The hydrophobic beads are organized in stripes. Surfactant-repelling surface beads surround these 
hydrophobic stripes. To quantify lateral confinement and possible cooperative behavior in our simulations we systematically changed the stripe width, L, and the shortest separation between parallel stripes, D. Note that surfactant-repelling surfaces such as those simulated here are probably not realizable experimentally, especially when the surfactants are in water. Despite this shortcoming, the model allows us to systematically investigate the effect of lateral confinement.

The simulations are intended to reproduce ambient conditions. Because DPD is a coarse-grained method, all simulation runs were conducted at the reduced temperature $\mathrm{k}_{\mathrm{B}} \mathrm{T}=1$. The random and dissipative parameters were set to $\sigma=3$ and $\gamma=4.5$, respectively. ${ }^{38}$ The time step $\Delta \mathrm{t}=0.03 \tau$ was used to integrate the equations of motion. The simulation time scale $\tau$ is 5.2 ps, as estimated by fitting the self-diffusion coefficient of water from our simulations for bulk water at ambient conditions to experimental data. Each system is simulated for as long as $~ 1.248 \mu \mathrm{s}$. By quantifying variations in the amount of surfactants present in the bulk supernatant (as micelles) and adsorbed on the surface, we found that equilibrium was established after $\sim 0.936 \mu$ s. Once in 1000 steps, the configurations were saved for analysis.

As the DPD method is based on soft interactions, the repulsion parameters that need to be chosen include intra-species $\mathrm{a}_{\mathrm{ii}}$ and inter-species $\mathrm{a}_{\mathrm{ij}}$ values. Following the protocol proposed by Groot and Warren, ${ }^{38} \mathrm{a}_{\mathrm{ii}}$ is derived from the compressibility of water $\left(\mathrm{a}_{\mathrm{ii}}=75 \mathrm{k}_{\mathrm{B}} \mathrm{T} / \rho\right)$. Because the simulation density is $\rho=5$ beads $/ \mathrm{r}_{\mathrm{c}}{ }^{3}$ and $\mathrm{k}_{\mathrm{B}} \mathrm{T}=1, \mathrm{a}_{\mathrm{ii}}=15$. The water-surfactant $\mathrm{a}_{\mathrm{ij}}$ parameters are borrowed from Kuo et al. ${ }^{43}$ However, note that the surfactant simulated here is composed by one head and two tail beads, while Kuo et al. represented sodium tetradecyl sulfate with only one head and one tail bead.

The choice of interaction parameters between water and surfactant molecules was validated by performing a series of bulk simulations to estimate critical micelle concentration (CMC) and micelle size. The formation of the first micelle was observed at $7.77 \mathrm{mM}$. Within a range of 
surfactant concentrations (up to $\sim 332 \mathrm{mM}$ ), our results for the micelle radius $(\sim 20.18 \AA$ ) were in general consistent with those reported in literature for SDS surfactants, both from experiments ${ }^{44-46}$ and from simulations. ${ }^{47-49}$ Observing the formation of micelles satisfied us, and we did not estimate the CMC from the free surfactant concentration, as proposed by Le Bard et al. ${ }^{50} \mathrm{We}$ were however surprised by observing only few free surfactants in equilibrium with micelles. Sanders et al. reported similar observations, ${ }^{51}$ from atomistic molecular dynamics simulations, in systems of strongly associating surfactants. As the surfactant concentration was increased to $443 \mathrm{mM}(\sim 57$ times the CMC) we observed the formation of cylindrical micelles.

The interactions between the surface beads and the surfactant tail beads were set to replicate tail-tail interactions when the surface bead is hydrophobic and water-tail interactions when the surface bead is repulsive. The interactions between the surface beads and the surfactant head beads were set to replicate tail-head interactions when the surface is hydrophobic and head-head interactions when the surface is repulsive. Implementing these parameters we observed the formation of a surfactant monolayer when a homogeneous hydrophobic surface was exposed to the aqueous surfactants. When the surfactant system was simulated on a homogeneous repelling surface, no surfactant adsorption was observed. We reiterate that this situation (a surface completely repelling surfactants) would be difficult to realize experimentally. In the latter case, all surfactants formed micelles in the bulk when the surfactant concentration is sufficiently high. Further details on the adsorption of surfactants on homogeneous surfaces are discussed in the next section. All interaction parameters implemented in this work are reported in Table 1.

The simulations were initiated with a surfactant concentration of $44.24 \mathrm{mM}$ (370 surfactant molecules). During the course of the simulations, as some surfactants adsorbed on the surfaces, additional surfactants were added to the systems in order to maintain constant concentration in the bulk. Such conditions are representative experimentally of systems where the amount of surfactant adsorbed is small compared to the total amount of surfactant present in the system, such as in 
ellipsometry $^{52,53}$ or quartz crystal microbalance ${ }^{13,26,54,55}$ experiments. The bulk concentration was maintained at $\sim 20-23 \mathrm{mM}(\approx 3 \mathrm{CMC})$. Some simulations were repeated at higher bulk concentration and yielded results similar to those presented below. Not surprisingly, simulation results below the $\mathrm{CMC}$ were however different, as the amount of surfactants adsorbed depends on the bulk surfactant concentration. The simulation results discussed within the manuscript are obtained from 68 individual simulations. The details for each of these simulations are provided in Table S1 of the Supporting Information.

\section{Results and Discussion}

\subsection{Adsorption on homogeneous surfaces}

We simulated surfactant adsorption and self-assembly on the two homogeneous solid surfaces, one hydrophobic and one surfactant repelling. The initial surfactant concentration was varied in the range $110.73-221.46 \mathrm{mM}(\approx 14.25-28.50 \mathrm{CMC})$, with all surfactants present in the bulk. On the hydrophobic surface, as expected, some surfactants adsorb as the simulation progresses, some form small aggregates in the bulk, and others remain as free monomers. Within a short period of time, however, the strong attractive interactions between the surfactant tail beads and the hydrophobic surface lead to complete adsorption. In Figure 2a we report an example of surfactants adsorption on the hydrophobic surface at the initial surfactant concentration of $141.70 \mathrm{mM}$. The hydrophobic surface is fully covered by surfactants at the surface coverage of $0.33 \mathrm{~nm}^{2}$ per surfactant molecule, yielding a flat monolayer. The surfactant tail groups are in contact with the hydrophobic surface, while the surfactant head groups are exposed to water. The excess surfactants form a spherical micelle in the bulk, where the surfactant concentration is at $7.77 \mathrm{mM}(\approx 1 \mathrm{CMC})$. The monolayer obtained in our simulations is expected, based on prior lattice Monte Carlo simulations for surfactants similar to those employed here. ${ }^{56}$ Because electrostatic interactions are not explicitly 
taken into consideration in our simulations, our results relate to experimental observations for nonionic surfactants on hydrophobic surfaces. ${ }^{57,58}$

In Figure $2 b$ we report a representative equilibrium simulation snapshot for the self-assembled surfactants when the surface is effectively repulsive. At the initial bulk concentration of $110.73 \mathrm{mM}$ employed, a number of spherical micelles are observed, but no surfactant is found to adsorb on the surface, as desired. However, no or at most only a few free surfactants are found, which is not representative of actual behavior, as briefly mentioned in the methods section.

\subsection{Adsorption on heterogeneous surfaces}

\section{A) Lateral confinement: Effect of stripe width}

To quantify the effect of lateral confinement on the properties of surfactant self-assembled aggregates we simulate surfactant adsorption on one individual hydrophobic stripe of variable width surrounded by repelling surface beads. By changing the stripe width we can manipulate the degree of lateral confinement, with narrow stripes yielding a more pronounced confinement that wide ones. The stripe width is indicated by $\mathrm{L}$, expressed in units of the length of one surfactant molecule, $\mathrm{L}_{\mathrm{s}}$. It is worth repeating that to maintain the thermodynamic conditions comparable throughout this study the surfactant concentration in the bulk, after adsorption has reached equilibrium, has been kept at $20-23 \mathrm{mM}(\approx 3 \mathrm{CMC})$. Representative simulation snapshots for surfactant aggregates adsorbed on one hydrophobic stripe of varying width are shown in Figure 3. From top to bottom the stripe width increases from $\mathrm{L}=0.15 \mathrm{~L}_{s}$, to $\mathrm{L}=1.68 \mathrm{~L}_{s}$. In the left and right panels we provide a view perpendicular to the axis of the surface plane and a side view, respectively. For clarity, water beads are not shown, nor are the surfactants present in the bulk, not adsorbed on the surface. These results should be compared with the simulation snapshot shown in Figure 2a, where the morphology is a flat monolayer with no lateral confinement. 
On the hydrophobic stripe of width $L=0.15 \mathrm{~L}_{s}$ (Figure $3 \mathrm{a}$ ) we sometimes observe the adsorption of one individual surfactant. When this surfactant is adsorbed, its tail beads are in contact with the hydrophobic stripe, while the head group protrudes towards water. Note that no bond angle is used in our model, hence the head bead is free to adopt various angles with respect to the surfactant tail. Besides this computational detail, and more important for the scope of the present manuscript is the observation that the single surfactant adsorbed on the narrow hydrophobic stripe frequently desorbs from it. Even increasing the surfactant concentration in the box to $110.73 \mathrm{mM}$ does not yield a permanently adsorbed surfactant aggregate for this system. Although the adsorption of one surfactant is possible, the formation of an aggregate containing more than one surfactant is not allowed with such a narrow stripe. This behavior is likely a consequence of the fact that advantageous tail-tail interactions between different surfactants cannot be established because the surrounding repelling surface prevents additional surfactants from adsorbing, and the surfactants adsorbed on the narrow hydrophobic stripe cannot adopt a conformation conducive to favorable tail-tail interactions.

The results obtained on the hydrophobic stripes of widths $L=0.31 \mathrm{~L}_{s}$, and $\mathrm{L}=0.46 \mathrm{~L}_{\mathrm{s}}$ are shown in Figure $3 b$, and $3 c$. The main difference compared to the data shown in Figure $3 a$ is the morphology of the self-assembled aggregate. On the narrowest stripe considered above one individual surfactant was found to adsorb, while on the stripes of width $\mathrm{L}=0.31 \mathrm{~L}_{\varepsilon}$ and $\mathrm{L}=0.46 \mathrm{~L}_{\varepsilon}$ the surfactants selfassemble yielding a hemi-sphere. As customary for such aggregates, the tail groups are found on the interior of the aggregate so they can be shielded from both water and the repelling surfaces. The surfactant headgroups are exposed to water, with some being positioned at the boundary between hydrophobic and repulsive surface beads. The self-assembled aggregates are found to easily migrate along the stripe. As the simulation progresses, similarly to the results discussed regarding the stripe of width $\mathrm{L}=0.15 \mathrm{~L}_{s}$, our results show that the surfactant aggregate can easily desorb from the stripe of width $\mathrm{L}=0.31 \mathrm{~L}_{s}$. On the stripe of width $\mathrm{L}=0.46 \mathrm{~L}_{s}$, however, once the aggregate forms on the 
surface, it does not desorb within the length of our simulations. The main driving force for surfactant adsorption is likely the attraction between surfactant tail groups and the hydrophobic stripe. The fact that the aggregates do not remain adsorbed for long times suggests that lateral confinement effectively reduces the effective attraction between the surfactants and the hydrophobic surface. The effective attraction is reduced compared to the homogeneous surface of Figure 2a because lateral confinement is so tight that it limits, to some extent, the cooperative tailtail interactions. These interactions are still present, as demonstrated by the formation of the adsorbed micelles, but they cannot be as extensive as they are on a flat homogeneous hydrophobic surface. Our observations seem to be in qualitative agreement with some experimental observations by Huang et al., ${ }^{59}$ who found that the adsorption of proteins on gold nanoparticles can be influenced by the scale of surface heterogeneity on the nanoparticles.

As the stripe width increases and becomes wider than half of the surfactant length, our simulation results reveal that the self-assembled aggregates change morphology from being roughly hemispherical to becoming hemi-cylindrical. These structures are shown in Figures $3 \mathrm{~d}, 3 \mathrm{e}$, and $3 \mathrm{f}$, for stripe widths $\mathrm{L}=0.61 \mathrm{~L}_{s}, 1.07 \mathrm{~L}_{s}$, and $1.68 \mathrm{~L}_{s}$, respectively. Other than the different shape of the aggregates, one important difference between the aggregates observed on these stripes and those obtained on the stripes of width $L=0.31 \mathrm{~L}_{s}$ or less is that once these aggregates are formed we do not observe desorption of the entire aggregate during the length of our simulations. Our results are consistent with prior simulations by others, who reported stable surfactant adsorption when the size of either checkerboard or striped surfaces is commensurate with the self-assembled surfactant aggregates. $^{28}$

As expected for surfactant aggregates with the hemi-cylindrical morphology self-assembled on hydrophobic surfaces, analysis of our simulation results confirms that the surfactant tail groups are adsorbed on the hydrophobic stripe, and are for the most part buried within the interiors of the hemi-cylindrical aggregate. The surfactant head groups provide a shield that separates the 
hydrophobic core of the hemi-cylindrical aggregates from the surrounding water. The average orientation of the surfactant molecules within these aggregates is perpendicular to the long axis of the hydrophobic stripes, as this orientation permits the surfactants to minimize hydrophobic surface - water and tail - water contacts, as well as to maximize the tail - tail interactions. Visual inspection of the simulation snapshots, in particular of the left panels of Figure 3 and shown closeup below in Figure 4, reveals that the surfactant head groups are in some cases just above the repelling surface beads. This result was not expected, as the interactions between surfactant headgroups and the repelling surface beads are somewhat repulsive (see Table 1). The energetic advantage of allowing numerous tail-tail and tail-hydrophobic surface interactions is compensating for the energy penalty related to having a few headgroups in proximity of the repelling surface beads.

Comparing the snapshots in Figure 4 (as well as those shown in Figure 3d, 3e, and 3f) we can observe that the curvature of the hemi-cylindrical surfactant aggregates decreases as the stripe width increases. On the stripe of width $\mathrm{L}=0.61 \mathrm{~L}_{s}$ (left panel), because the surfactant molecules cannot extend up to their length while adsorbed on the hydrophobic surface, a few of them align perpendicular to the surface, yielding a hemi-cylinder with large curvature. On the stripe of width $\mathrm{L}$ $=1.07 \mathrm{~L}_{s}$ (middle panel) the surfactant molecules preferentially orient themselves perpendicular to the direction of the long axis of the stripe, similarly to the results obtained for the stripe of width $\mathrm{L}$ $=1.68 \mathrm{~L}_{s}$ (right panel). On a much wider stripe, i.e., on a homogenous hydrophobic surface, the surfactants considered here yield a monolayer, as shown in Figure 2a. Combining all these sets of visual observations, our results suggest that lateral confinement effectively compresses the monolayer laterally, eventually making it bulge away from the surface. As the lateral confinement becomes of the order of the surfactants molecular length, the monolayer becomes a hemi-cylinder. When the lateral confinement is even more pronounced, the hemi-cylinder is no longer stable, and 
therefore desorbs, allowing some surfactants to adsorb as hemi-spheres, and eventually preventing aggregates from forming on the surface.

We also calculated in-plane density profiles of surfactant tail groups, and surfactant head groups within planes parallel (top panels) and perpendicular (bottom panels) to the surface, as presented in Figures 5, and 6, respectively. From left to right we show the results at increasing stripe width. We only consider stripes of width $\mathrm{L}=0.61 \mathrm{~L}_{\varepsilon}$ or wider, as in the narrower stripes the aggregates are mobile and the density profiles would provide a misleading characterization.

The main observation from visual inspection of the results, shown in Figure 5, is that the surfactant tail groups are concentrated near the center of the stripes. On the stripe of width $\mathrm{L}=0.61 \mathrm{~L}_{s}$ the surfactant tail groups are effectively curved out at the position of the stripe edges. When the stripe width increases, the surfactant tail groups are less present at the boundary between hydrophobic stripe and repelling surface. In Figure 6 the results confirm the analysis of the snapshots of Figure 4, in that the curvature of the hemi-cylindrical surfactant aggregates clearly decreases as the stripe width increases. The surfactant head groups evidently accumulate above the repelling surface beads on the three stripes, especially in the case of the stripe of width $\mathrm{L}=0.61 \mathrm{~L}_{s}$, most likely because assuming such conformation the surfactants benefit from advantageous tailgroup-hydrophobic surface interactions. On the stripe of width $\mathrm{L}=1.68 \mathrm{~L}_{s}$, the surfactant head groups spread, and near the center of the stripe yield a structure comparable to that of a monolayer. These effects are a manifestation of the lateral confinement, as discussed above. The results in Figure 5 and 6 are in qualitative analogy with the observation reported by Tummala et al., ${ }^{30}$ who conducted atomistic molecular dynamics simulations and reported that the size of a graphene sheet can strongly affect the morphology of a self-assembled SDS aggregate, presumably because of lateral confinement effects. 


\section{B) Cooperative effects: Adsorption on neighboring stripes}

Cooperative effects have been investigated by simulating the self-assembly of surfactants on two stripes, of variable width, at different separations from each other. The shortest separation between the two stripes is indicated by the symbol D, expressed in units of the length of one surfactant molecule, $\mathrm{L}_{\mathrm{s}}$. In Figure 7 we provide representative simulation snapshots of surfactant aggregates adsorbed on two stripes separated by $\mathrm{D}=0.15 \mathrm{~L}_{s}$ (top panels) and $0.46 \mathrm{~L}_{s}$ (bottom panels). In all cases considered in this figure the hydrophobic stripes are of width $\mathrm{L}=0.46 \mathrm{~L}_{\mathrm{s}}$. In the left and right panels we provide a view perpendicular to the axis of the surface plane, a side view, and a close-up snapshot for the surfactant aggregates, respectively. It should be noted that on an individual stripe of width $L=0.46 \mathrm{~L}_{s}$ the surfactants adsorb yielding hemi-spheres (see Figure $3 \mathrm{c}$ ). When the two narrow stripes are separated but close to each other (all cases in Figure 7) our simulations reveal the formation of a roughly hemi-cylindrical shell. The surfactant tail groups are adsorbed on the hydrophobic stripe, and are assembled into a monolayer that is curved in the middle so that the hydrophobic tails do not contact the repulsive surface. Within this shell the surfactant head groups are exposed to water towards the outside of the hemi-cylinder, while some of the tail groups, in the center of the shell, are exposed to the surfactant-repelling surface in between the two hydrophobic stripes. The two parallel hydrophobic stripes essentially provide a support for this monolayer. Our results show no evidence of water inside the hemi-cylinders. The self-assembled shells have the features just summarized for two reasons: (1) the surfactants are repelled by the surface separating the two hydrophobic stripes, and, perhaps more importantly, (2) bending of the monolayer allows for the formation of numerous tail-tail contacts at the expense of an elastic deformation of the monolayer (which we did not quantify). By changing the molecular properties of the surfactants, the curvature of the hemi-cylindrical shell might be controlled, which could lead to advancements in manufacturing processes and surface modification techniques. Because our results show that the self-assembled surfactant monolayer effectively covers the repelling region that separates the two 
hydrophobic surfaces, our simulations appear to be consistent with the MC simulation results of Reimer et al., ${ }^{19}$ who studied surfactant adsorption on checkerboard surfaces. Surfactant adsorption was enhanced when the attractive patches were sufficiently close on an otherwise repelling surface.

To further quantify the results just summarized, in Figure 8 we present planar density distributions obtained for surfactant tail groups (top panels) and head groups (bottom panels) as observed along planes perpendicular to the surface. From left to right we provide the results obtained for the separation distance $D=0.15 \mathrm{~L}_{s}$ and $0.46 \mathrm{~L}_{s}$, respectively. Confirming the visual inspection of the simulation snapshots of Figure 7, the density profiles show that the surfactant tail groups form a dense layer between the two neighboring hydrophobic stripes, and the head groups are exposed only to the outside of the hemi-cylinder. Next we attempt to quantify the effect of increasing D on the results just discussed.

Representative simulation snapshots of the surfactant aggregates obtained at increasing D are shown in Figure 9. For these simulations $L=0.46 \mathrm{~L}_{s}$. As discussed in Figure 7, when the two hydrophobic stripes are parallel and close to each other we obtained hemi-cylindrical shells. At intermediate separations we observe that the hemi-cylindrical aggregate is spoiled. At $\mathrm{D}=0.76 \mathrm{~L}_{s}$ our results suggest that the hemi-cylindrical shell is partially formed, but it does not extend to the entire length of the simulation box. In addition, elongated hemi-spheres form on each of the two hydrophobic stripes. As $\mathrm{D}$ further increases to $\mathrm{D}=1.07 \mathrm{~L}_{\mathrm{s}}$ the hemi-cylindrical shell can no longer form (most likely because of entopic effects that prevent the formation of an extended partially unsupported monolayer suspended between the two narrow hydrophobic stripes). In addition, the aggregates that form on each hydrophobic stripe appear to be more elongated and irregular than the hemi-spheres discussed in Figure 3c. Visual analysis of sequences of simulation snapshots suggests that the aggregates formed at these conditions are very flexible and more freely along the hydrophobic stripes. This property of the self-assembled aggregates suggest the possibility that aggregates such as those shown in Figure 9 might be responsible for the high energy dissipation reported for quartz 
crystal microbalance experimental measurements by $\mathrm{Wu}$ et al. $^{26}$ when surfactants adsorbed on molecularly rough surfaces at conditions near the surfactants CMC. No evidence of cooperative effect was observed in our simulations when $\mathrm{D}$ was increased to $1.38 \mathrm{~L}_{s}$ or above; the surfactant aggregates self-assembled on each hydrophobic stripe yield the hemi-spheres shown in Figure 3c.

Next we consider cooperative effects on hydrophobic stripes wider than those considered for the simulations of Figures 7, 8, and 9. In Figure 10 we present representative simulation snapshots of surfactant aggregates adsorbed on two stripes of width $\mathrm{L}=1.07 \mathrm{~L}_{s}$ (top panels) and $\mathrm{L}=1.68 \mathrm{~L}_{s}$ (bottom panels) at the separation $\mathrm{D}=0.15 \mathrm{~L}_{s}$. Although the two hydrophobic stripes are close to each other for each of the systems considered in this figure, our results show that the surfactants yield hemi-cylinders analogous to those obtained on the individual hydrophobic stripes of width comparable to those considered here (compare the snapshots in Figure 10 to those shown in Figure 3 , bottom panels). Because the stripes considered in these simulations are wide with respect to the molecular length of one surfactant, the surfactant molecules do not find it advantageous to alter the stable structures discussed in Figures 3, 4, 5, and 6, even though two hydrophobic stripes are close to each other. Hemi-cylinders allow the surfactant molecules to maximize the favorable energetic interactions between the hydrophobic stripe and the tail groups, as well as those between those the various surfactant beads within the aggregates. As a consequence, our simulations do not show cooperative effects when the stripes are large enough for the formation of energetically stable aggregates.

\section{Conclusions}

The adsorption of surfactants on flat heterogeneous surfaces was studied by performing equilibrium dissipative particle dynamics (DPD) simulations. Surfactant molecules were modeled as having a hydrophobic tail composed by two beads and a hydrophilic head composed by one bead. The 
surface had one or two hydrophobic stripes on which the surfactants can adsorb with the remainder of the surface repelling the surfactants. To understand the effect of surface heterogeneity on surfactant adsorption, the stripe width and the separation distance between two parallel stripes were varied systematically in the different simulations presented. For all systems, a sufficient amount of surfactant was present to maintain a bulk concentration above the critical micelle concentration after adsorption was completed. The surfactants yield a flat monolayer on a homogeneous hydrophobic surface and did not adsorb on the homogeneous repelling surface. Flat monolayers, hemi-cylinders, hemi-spheres, and individual surfactants were obtained as the width of the hydrophobic stripe decreased. Our results showed cooperative effects when two adsorbing stripes were separated, yet close to each other, but only when the stripes were narrower than the length of one surfactant molecule. Hemi-cylindrical shells and irregular structures were observed, as evidence of cooperativity. No evidence for cooperative behavior was found when the stripes were close to each other, but wide with respect to the surfactant length, or when the narrow parallel stripes were farther than $\sim 1.07$ times the length of one surfactant molecule.

\section{Acknowledgments}

The authors acknowledge financial support from the industrial sponsors of the Institute for Applied Surfactants Research (IASR) at the University of Oklahoma and from the US National Science Foundation (NSF) under Grant No. CMMI-1068705. The sponsoring companies of the Institute for Applied Surfactant Research are: CESI Chemical, Church \& Dwight, Clorox, ConocoPhillips, Ecolab, GlaxoSmithKline, Halliburton Services, Huntsman, InVia-WestVaco, Novus, Procter and Gamble, Sasol, S.C. Johnson, and Shell Chemical. AS and MS wish to thank the Department of Chemical Engineering at the University College London, for financially supporting, in part, this research. Allocations of computing time were provided by the OU Supercomputing Center for Education and Research (OSCER) at the 
University of Oklahoma, and by the National Energy Research Scientific Computing Center (NERSC) at Lawrence Berkeley National Laboratory, Berkeley, CA.

\section{References}

1. R. Butler, I. Hopkinson and A. I. Cooper, J. Am. Chem. Soc., 2003, 125, 14473-14481.

2. M. L. Campbell, D. L. Apodaca, M. Z. Yates, T. M. McCleskey and E. R. Birnbaum, Langmuir, 2001, 17, 5458-5463.

3. K. Ghosh, S. M. Vyas, H. J. Lehmler, S. E. Rankin and B. L. Knutson, J. Phys. Chem. B, 2007, 111, 363-370.

4. J. P. Hanrahan, M. P. Copley, K. M. Ryan, T. R. Spalding, M. A. Morris and J. D. Holmes, Chem. Mat., 2004, 16, 424-427.

5. S. Paria and K. C. Khilar, Adv. Colloid Interface Sci., 2004, 110, 75-95.

6. I. U. Vakarelski, S. C. Brown, Y. I. Rabinovich and B. M. Moudgil, Langmuir, 2004, 20, 1724-1731.

7. K. M. K. Yu, A. M. Steele, J. Zhu, Q. J. Fu and S. C. Tsang, J. Mater. Chem., 2003, 13, 130-134.

8. F. Tiberg, B. Jonsson and B. Lindman, Langmuir, 1994, 10, 3714-3722.

9. F. Tiberg, B. Jonsson, J. Tang and B. Lindman, Langmuir, 1994, 10, 2294-2300.

10. R. Atkin, V. S. J. Craig, E. J. Wanless and S. Biggs, Adv. Colloid Interface Sci., 2003, 103, 219-304.

11. S. Manne and H. E. Gaub, Science, 1995, 270, 1480-1482.

12. S. Manne, J. P. Cleveland, H. E. Gaub, G. D. Stucky and P. K. Hansma, Langmuir, 1994, 10, 4409-4413.

13. P. M. Karlsson, A. E. C. Palmqvist and K. Holmberg, Langmuir, 2008, 24, 13414-13419.

14. S. O. Nielsen, G. Srinivas, C. F. Lopez and M. L. Klein, Phys. Rev. Lett., 2005, 94, 4. 
15. K. Shah, P. Chiu and S. B. Sinnott, J. Colloid Interface Sci., 2006, 296, 342-349.

16. T. Zehl, A. Wahab, P. Schiller and H. J. Mogel, Langmuir, 2009, 25, 2090-2100.

17. Z. J. Xu, X. N. Yang and Z. Yang, J. Phys. Chem. B, 2008, 112, 13802-13811.

18. X. R. Zhang, B. H. Chen and Z. H. Wang, J. Colloid Interface Sci., 2007, 313, 414-422.

19. U. Reimer, M. Wahab, P. Schiller and H. J. Mogel, Langmuir, 2001, 17, 8444-8450.

20. H. Dominguez, A. G. Goicochea, N. Mendoza and J. Alejandre, J. Colloid Interface Sci., 2006, 297, 370-373.

21. K. Shah, P. Chiu, M. Jain, J. Fortes, B. Moudgil and S. Sinnott, Langmuir, 2005, 21, 53375342.

22. G. Srinivas, S. O. Nielsen, P. B. Moore and M. L. Klein, J. Am. Chem. Soc., 2006, 128, 848853.

23. J. Foisner, A. Glaser, T. Leitner, H. Hoffmann and G. Friedbacher, Langmuir, 2004, 20, 2701-2706.

24. H. C. Schniepp, H. C. Shum, D. A. Saville and I. A. Aksay, J. Phys. Chem. B, 2007, 111, 8708-8712.

25. H. C. Schniepp, H. C. Shum, D. A. Saville and I. A. Aksay, J. Phys. Chem. C, 2008, 112, 14902-14906.

26. S. Wu, L. Shi, L. B. Garfield, R. F. Tabor, A. Striolo and B. P. Grady, Langmuir, 2011, 27, 6091-6098.

27. U. Reimer, M. Wahab, P. Schiller and H. J. Mogel, Langmuir, 2005, 21, 1640-1646.

28. X. R. Zhang, B. H. Chen, W. Dong and W. C. Wang, Langmuir, 2007, 23, 7433-7435.

29. A. Striolo, J. Chem. Phys., 2006, 125, 9.

30. N. R. Tummala, B. P. Grady and A. Striolo, Phys. Chem. Chem. Phys., 2010, 12, 1313713143.

31. M. Suttipong, N. R. Tummala, B. Kitiyanan and A. Striolo, J. Phys. Chem. C, 2011, 115, 17286-17296. 
32. N. R. Tummala, B. H. Morrow, D. E. Resasco and A. Striolo, ACS Nano, 2010, 4, 71937204.

33. N. R. Tummala and A. Striolo, ACS Nano, 2009, 3, 595-602.

34. M. Suttipong, N. R. Tummala, A. Striolo, C. S. Batista and J. Fagan, Soft Matter, 2013, 9, 3712-3719.

35. S. Plimpton, J. Comput. Phys., 1995, 117, 1-19.

36. E. Moeendarbary, T. Y. Ng and M. Zangeneh, Int. J. Appl. Mech., 2009, 1, 737-763.

37. R. D. Groot and K. L. Rabone, Biophys. J., 2001, 81, 725-736.

38. R. D. Groot and P. B. Warren, J. Chem. Phys., 1997, 107, 4423-4435.

39. J. R. Lu, Z. X. Li, R. K. Thomas, E. J. Staples, I. Tucker and J. Penfold, J. Phys. Chem., 1993, 97, 8012-8020.

40. R. Nagarajan and C. C. Wang, Langmuir, 2000, 16, 5242-5251.

41. H. Zhao and K. L. Nagy, J. Colloid Interface Sci., 2004, 274, 613-624.

42. N. Denham, M. C. Holmes and A. V. Zvelindovsky, J. Phys. Chem. B, 2011, 115, 13851393.

43. M. Y. Kuo, H. C. Yang, C. Y. Hua, C. L. Chen, S. Z. Mao, F. Deng, H. H. Wang and Y. R. Du, ChemPhysChem, 2004, 5, 575-580.

44. K. J. Mysels, Langmuir, 1986, 2, 423-428.

45. P. H. Elworthy and K. J. Mysels, J. Colloid Interface Sci., 1966, 21, 331-\&.

46. B. Cabane, R. Duplessix and T. Zemb, J. Phys., 1985, 46, 2161-2178.

47. S. Jalili and M. Akhavan, Colloid Surf. A-Physicochem. Eng. Asp., 2009, 352, 99-102.

48. C. D. Bruce, M. L. Berkowitz, L. Perera and M. D. E. Forbes, J. Phys. Chem. B, 2002, 106, 3788-3793.

49. A. D. Mackerell, J. Phys. Chem., 1995, 99, 1846-1855.

50. D. N. LeBard, B. G. Levine, P. Mertmann, S. A. Barr, A. Jusufi, S. Sanders, M. L. Klein and A. Z. Panagiotopoulos, Soft Matter, 2012, 8, 2385-2397. 
51. S. A. Sanders, M. Sammalkorpi and A. Z. Panagiotopoulos, J. Phys. Chem. B, 2012, 116, 2430-2437.

52. F. Tiberg, B. Joensson, J.-a. Tang and B. Lindman, Langmuir, 1994, 10, 2294-2300.

53. F. Tiberg, B. Joesson and B. Lindman, Langmuir, 1994, 10, 3714-3722.

54. C. Gutig, B. P. Grady and A. Striolo, Langmuir, 2008, 24, 4806-4816.

55. D. Johannsmann, I. Reviakine, E. Rojas and M. Gallego, Anal. Chem., 2008, 80, 8891-8899.

56. F. X. Zheng, X. R. Zhang, W. C. Wang and W. Dong, Langmuir, 2006, 22, 11214-11223.

57. L. M. Grant, T. Ederth and F. Tiberg, Langmuir, 2000, 16, 2285-2291.

58. L. M. Grant and W. A. Ducker, J. Phys. Chem. B, 1997, 101, 5337-5345.

59. R. Huang, R. P. Carney, F. Stellacci and B. L. T. Lau, Nanoscale, 2013, 5, 6928-6935. 
Table 1. Repulsion parameters $\mathrm{a}_{\mathrm{ii}}$ and $\mathrm{a}_{\mathrm{ij}}$, for water $(\mathrm{W})$, surfactant headgroup $(\mathrm{H})$, surfactant tail (T), surfactant-repelling surface (RS), and hydrophobic (adsorbing) surface (AS) beads.

\begin{tabular}{cccccc} 
& W & H & T & RS & AS \\
W & 15 & 0 & 81 & 15 & 81 \\
H & & 15 & 69 & 15 & 69 \\
T & & & 15 & 81 & 15 \\
RS & & & & 15 & 81 \\
AS & & & & & 15 \\
\hline
\end{tabular}


(a)

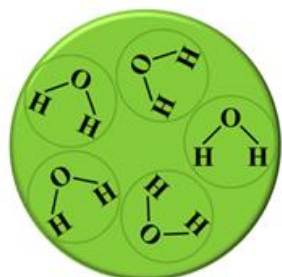

Water Bead

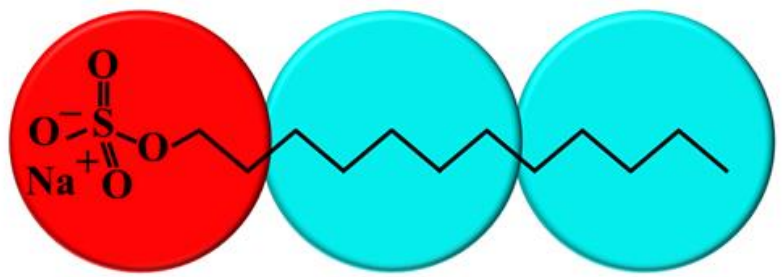

Surfactant Molecule

(b)

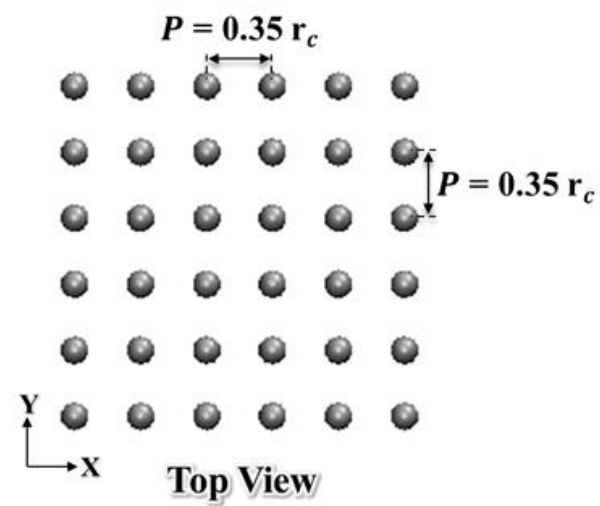

(c)

Side View
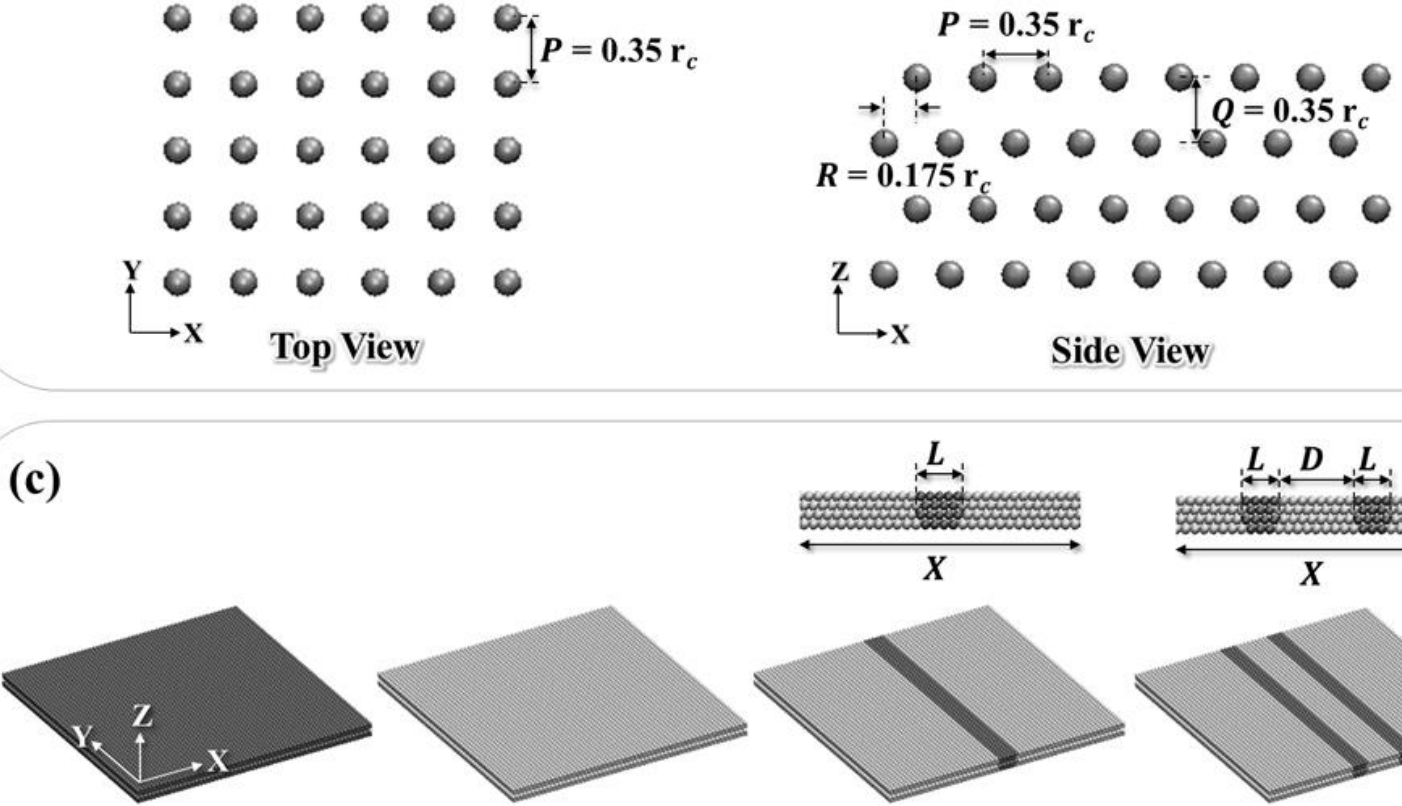

Hydrophobic Surface

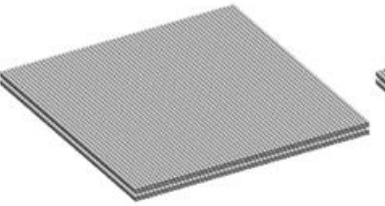

Surfactant-Repelling Surface
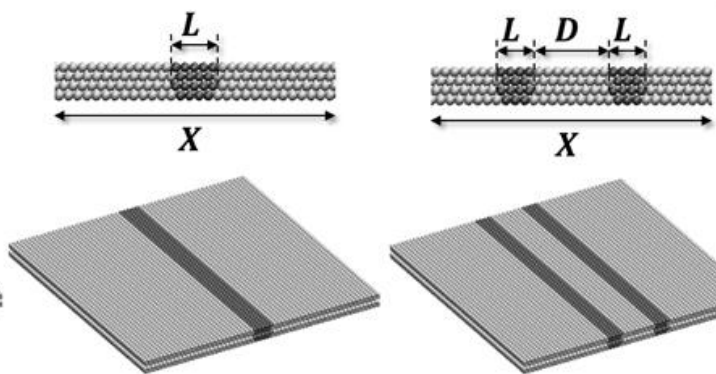

One Stripe

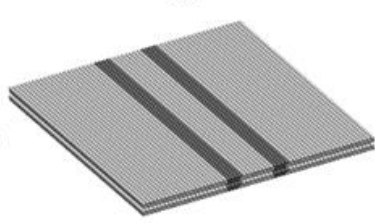

Two Stripes

Figure 1. Schematic representation of (a) the coarse-grained beads used to represent water and surfactant molecules. The DPD water bead (green) represents five water molecules. The surfactant, which mimics SDS, contains one headgroup bead (red sphere) and two tail beads (cyan spheres). (b) Top (left) and side (right) views of the solid surface, highlighting the position of the rigid beads. The surface beads in each layer are arranged in a square lattice in which $\mathbf{P}$ is the nearest-neighbor distance along both $\mathrm{X}$ and $\mathrm{Y}$ directions. $\mathrm{Q}$ is the interlayer distance between planes of atoms within the solid. $\mathbf{R}$ is the shift distance between neighboring surface planes. (c) Patterned flat surfaces employed in this study. The hydrophobic and the surfactant-repelling surfaces are shown as dark 
and light gray, respectively. $\mathrm{L}$ is the stripe width. $\mathrm{D}$ is the shortest separation distance between two stripes.

(a)

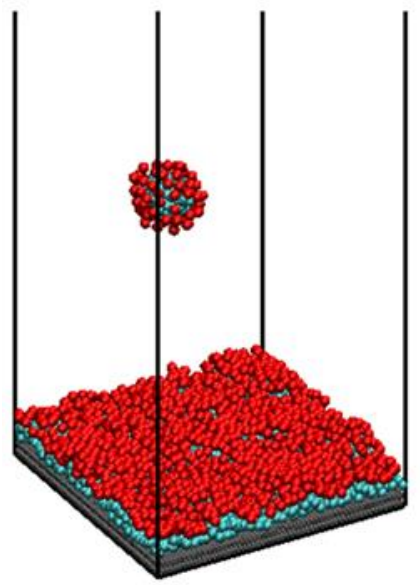

(b)

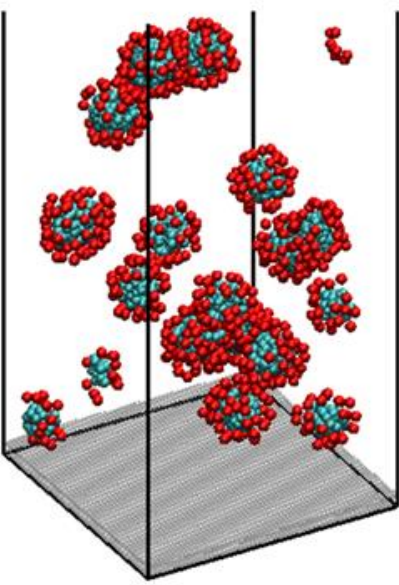

Figure 2. Representative simulation snapshots representing self-assembled surfactant aggregates on (a) the hydrophobic and (b) the surfactant-repelling surfaces. The simulations are conducted with initial surfactant concentrations of 141.70 and $110.73 \mathrm{mM}$, respectively. Surfactant head and tail groups are shown as red and cyan spheres, respectively. The hydrophobic and the surfactantrepelling surfaces are shown as dark and light gray, respectively. Water beads are not shown for clarity. 
(a)
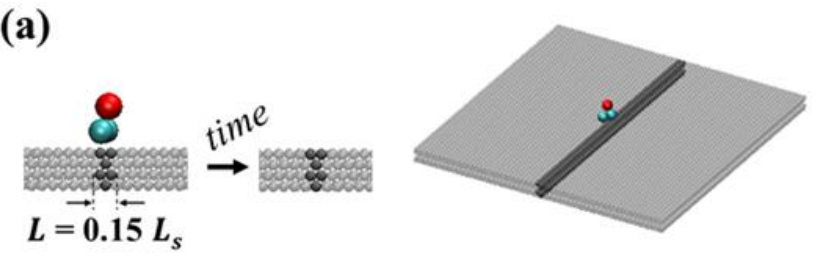

(b)
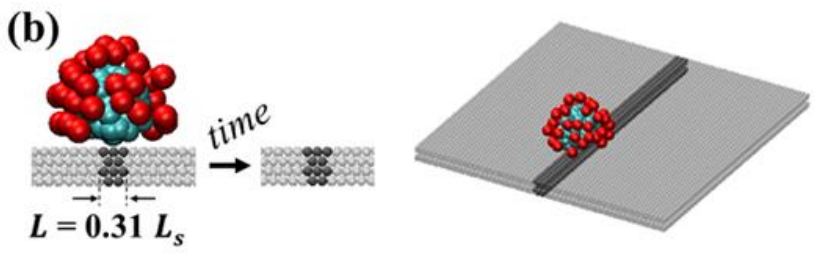

(c)
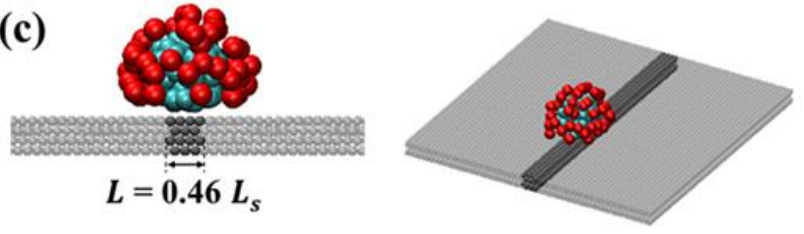

(d)
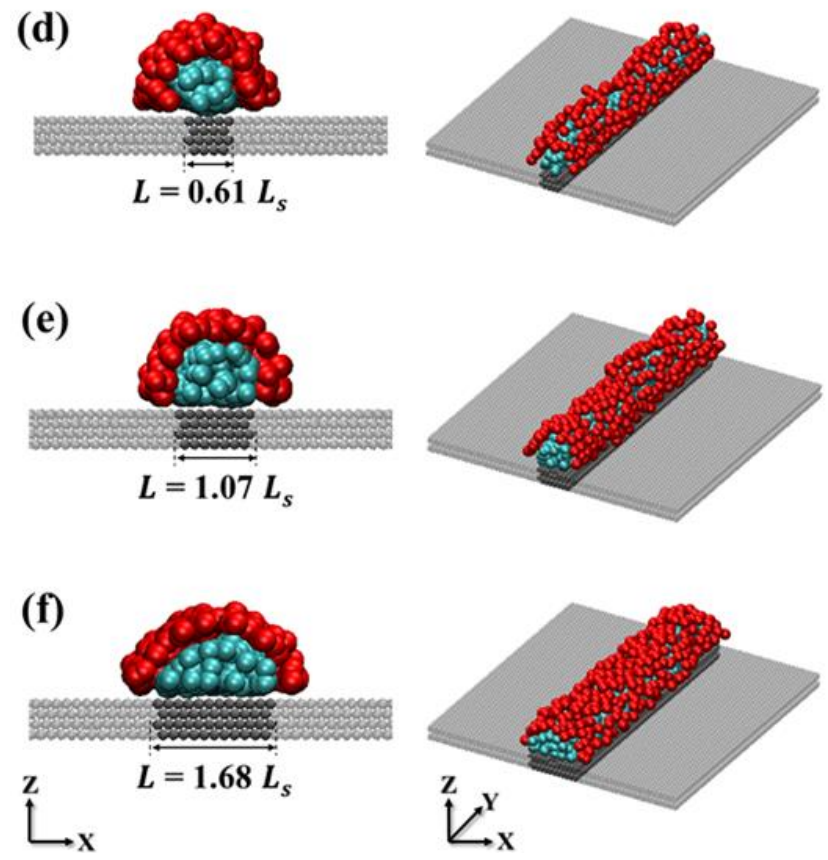

Figure 3. Surfactant aggregates self-assembled on one hydrophobic stripe of width $L=$ (a) $0.15 L_{s}$; (b) $0.31 \mathrm{~L}_{s}$; (c) $0.46 \mathrm{~L}_{s}$; (d) $0.61 \mathrm{~L}_{s}$; (e) $1.07 \mathrm{~L}_{s}$; and (f) $1.68 \mathrm{~L}_{s}$. Left and right panels are for a view perpendicular to the axis of the surface plane and a side view, respectively. Water beads are not shown for clarity. The color code is the same as that used in Figure 2. Note that for systems (a) and (b) the aggregates adsorb and desorb frequently from the hydrophobic stripe, as discussed in the text. 

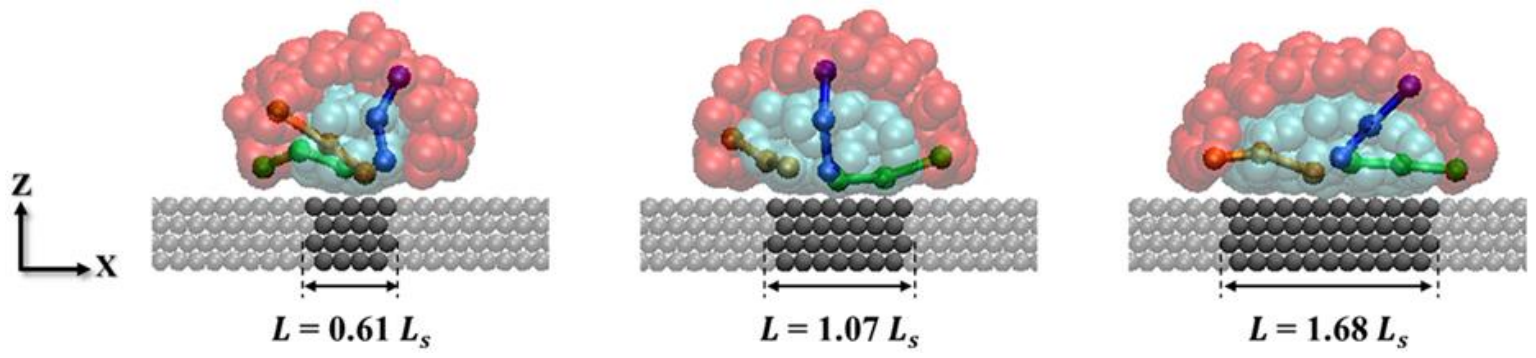

Figure 4. Close-up snapshots of surfactant aggregates self-assembled on hydrophobic stripes of width $\mathrm{L}=0.61 \mathrm{~L}_{s}, 1.07 \mathrm{~L}_{s}$, and $1.68 \mathrm{~L}_{s}$ (left to right panels, respectively). In this figure is shown a view perpendicular to the axis of the surface plane. Water beads are not shown for clarity. The color code is the same as that used in Figure 2.

(a)
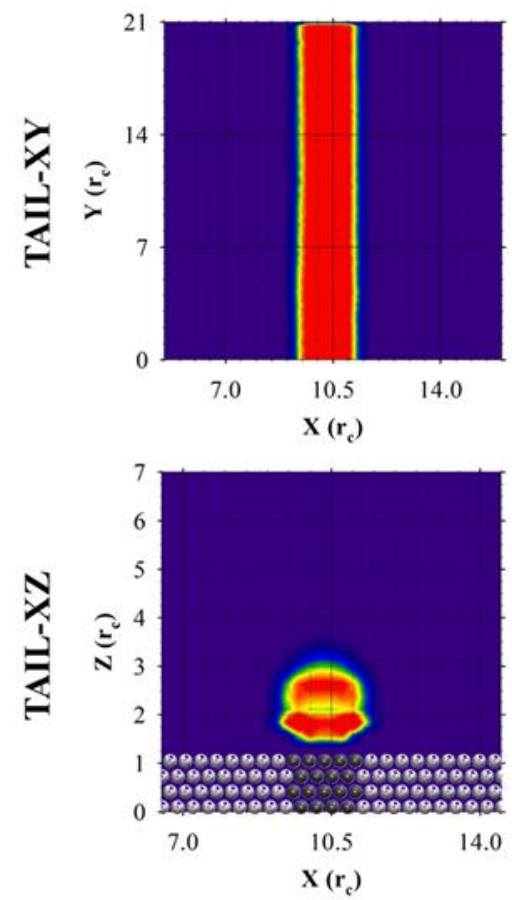

(b)
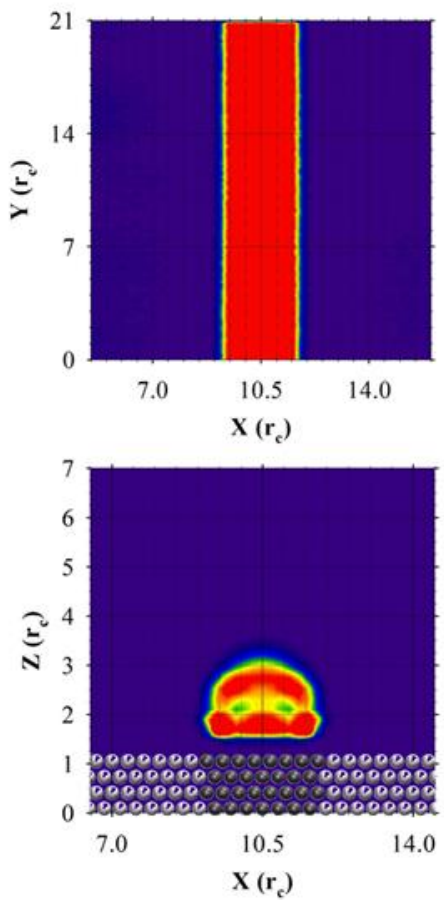

(c)
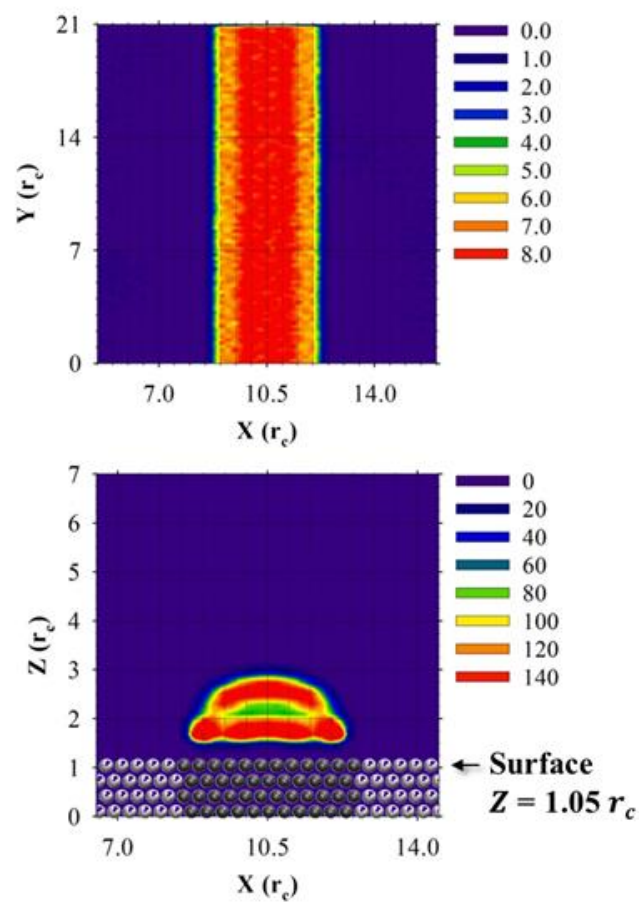

Figure 5. In-plane density distributions of surfactant tail groups adsorbed on one individual hydrophobic stripe. The results are obtained along planes parallel (top) and perpendicular (bottom) to the surface. Left to right panels are for stripes of width $\mathrm{L}=0.61 \mathrm{~L}_{s}, 1.07 \mathrm{~L}_{s}$, and $1.68 \mathrm{~L}_{s}$, respectively. Surface densities are expressed in number of beads per $r_{c}{ }^{2}$. 
(a)
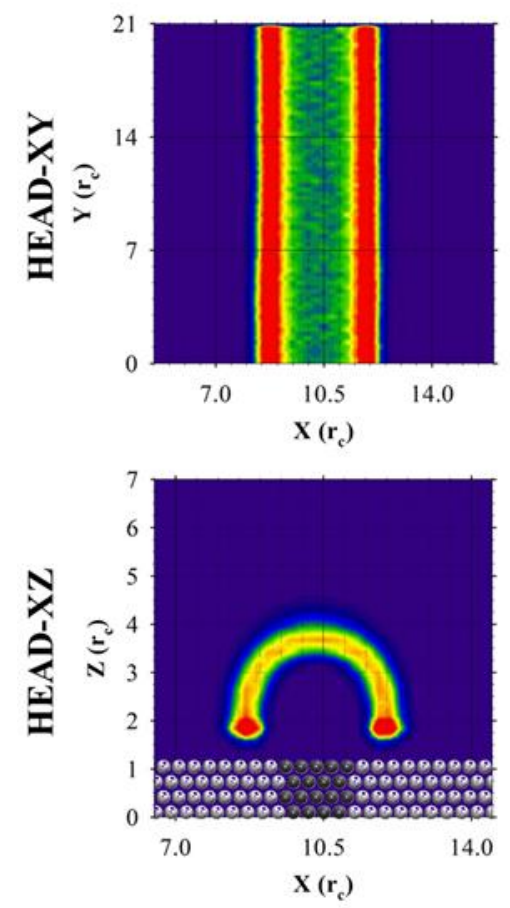

(b)
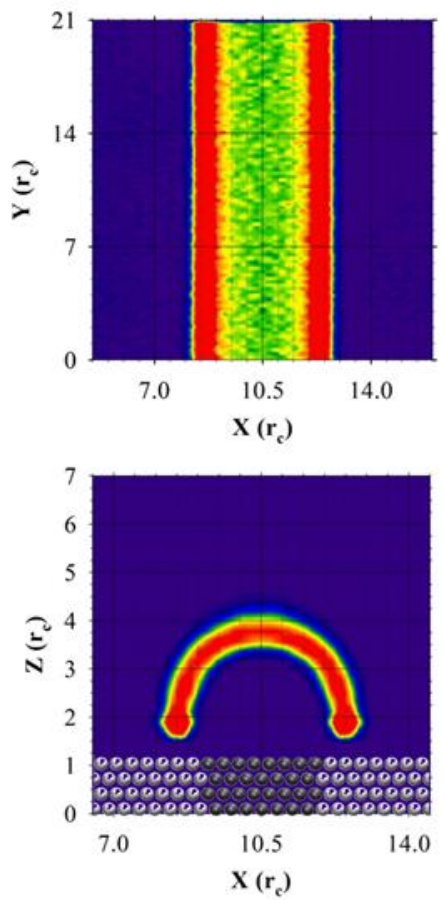

(c)
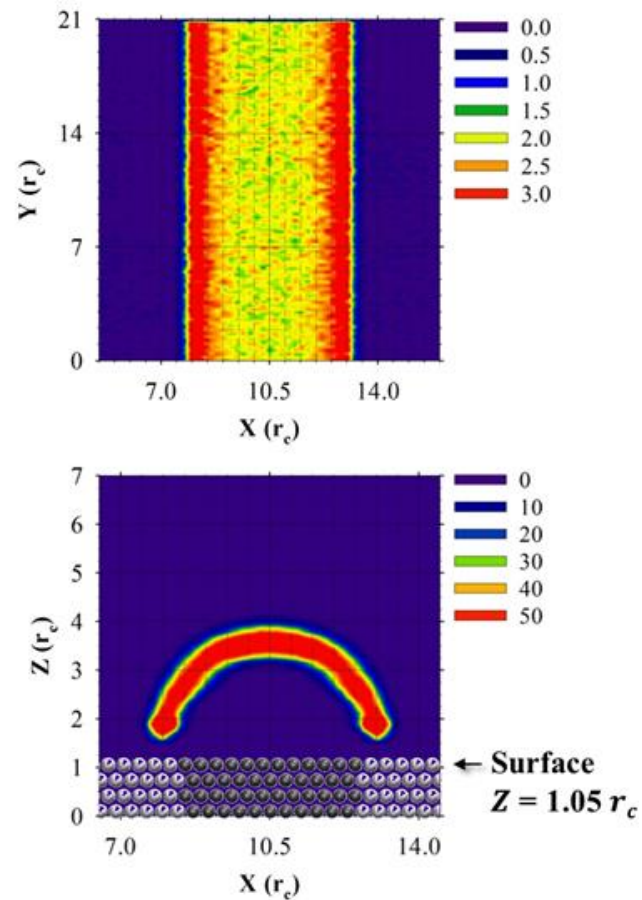

Figure 6. In-plane density distributions of surfactant head groups adsorbed on one individual hydrophobic stripe. The results are obtained along planes parallel (top) and perpendicular (bottom) to the surface. Left to right panels are for the stripes of width $\mathrm{L}=0.61 \mathrm{~L}_{s}, 1.07 \mathrm{~L}_{s}$, and $1.68 \mathrm{~L}_{s}$, respectively. Surface densities are expressed in number of beads per $r_{c}{ }^{2}$. 

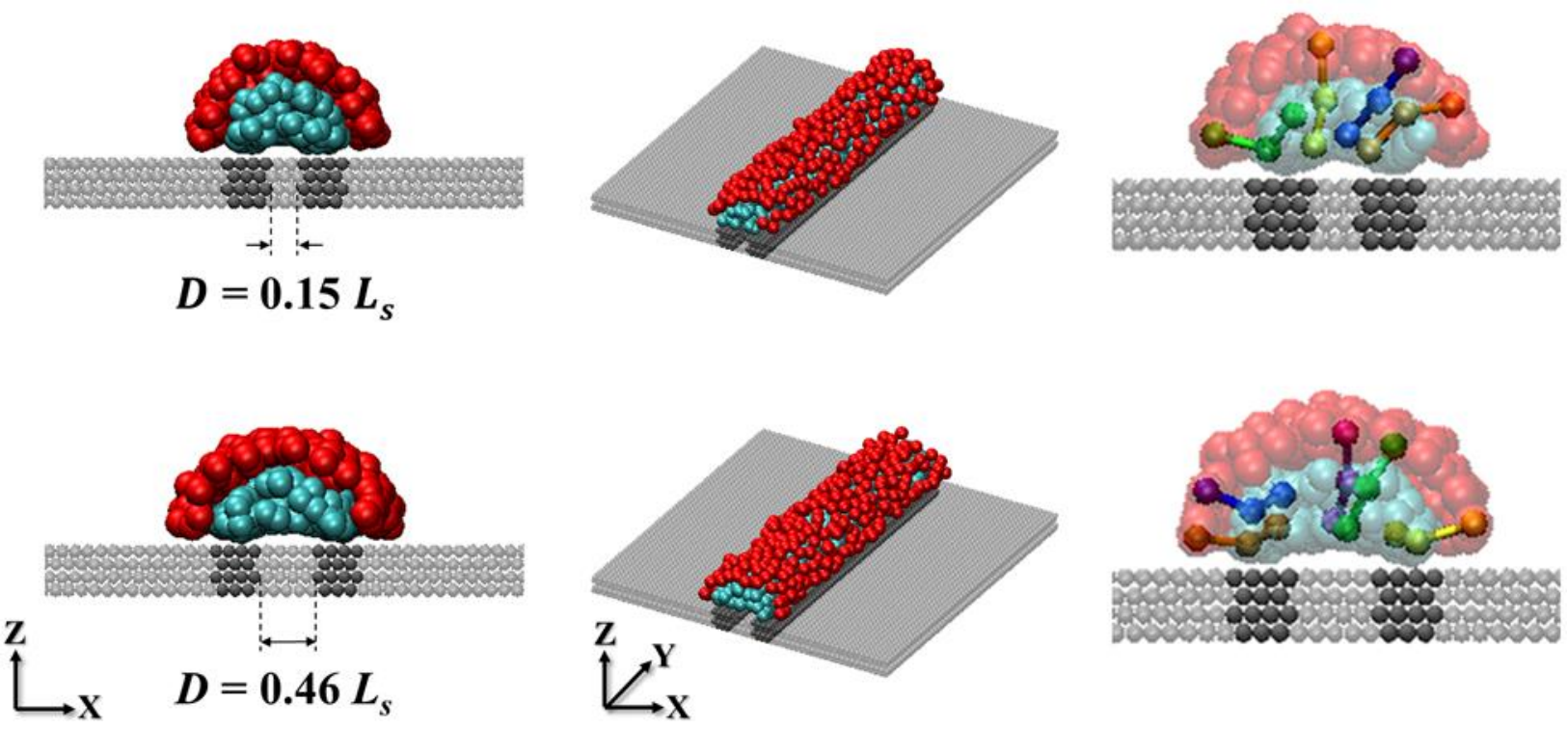

Figure 7. Representative simulation snapshots for surfactant aggregates self-assembled on two hydrophobic stripes located at minimum separation distance $\mathrm{D}=0.15 \mathrm{~L}_{s}$ (top), and $0.46 \mathrm{~L}_{s}$ (bottom). The results are for stripes of width $\mathrm{L}=0.46 \mathrm{~L}_{\mathrm{s}}$. Left and right panels are for a view perpendicular to the axis of the surface plane, a side view, and a close-up snapshot of surfactant aggregates, respectively. Water beads are not shown for clarity. The color code is the same as that used in Figure 2. 

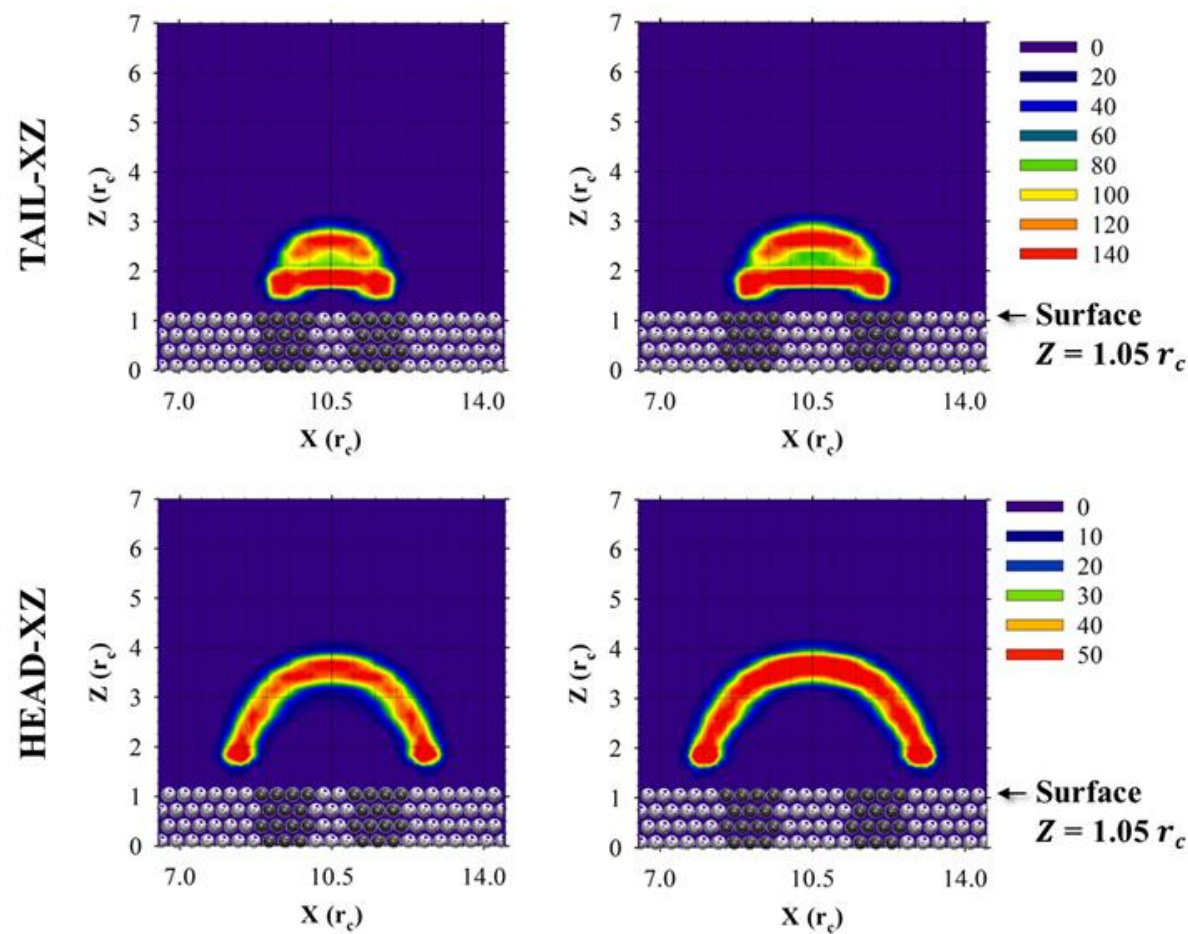

Figure 8. In-plane density distributions of surfactant tail groups (top) and head groups (bottom) for surfactants adsorbed on two hydrophobic stripes located at minimum separation distances $\mathrm{D}=$ $0.15 \mathrm{~L}_{s}$ and $0.46 \mathrm{~L}_{s}$ (left and right panels, respectively). The results are obtained for stripes of width $\mathrm{L}=0.46 \mathrm{~L}_{\mathrm{s}}$ along a plane perpendicular to the surface. Surface densities are expressed as number beads per $\mathrm{r}_{\mathrm{c}}{ }^{2}$. 


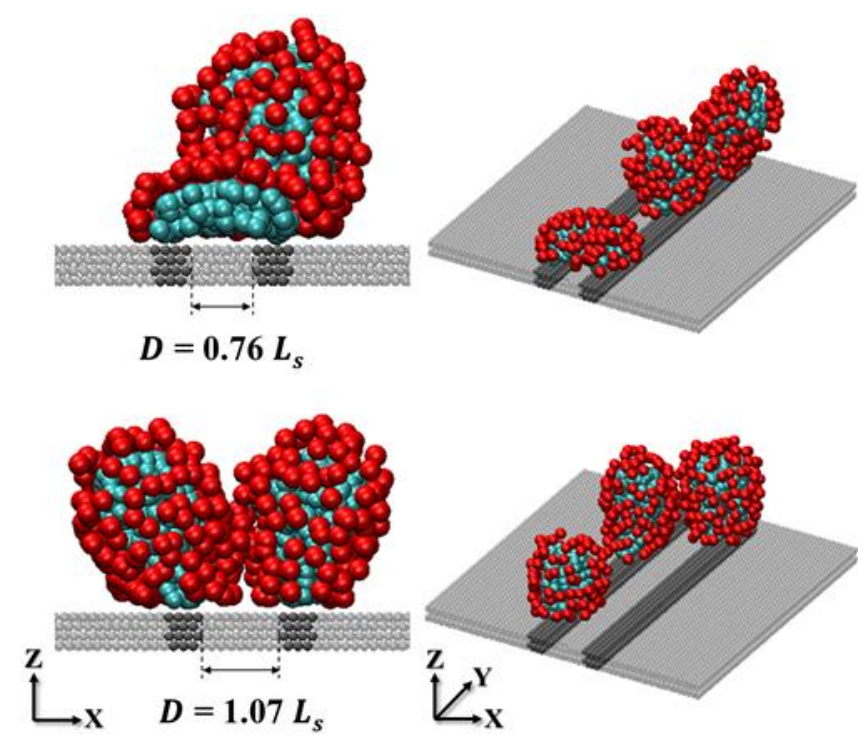

Figure 9. Representative simulation snapshots for surfactant aggregates self-assembled on two hydrophobic stripes located at minimum separation distances $\mathrm{D}=0.76 \mathrm{~L}_{s}$ (top) or $1.07 \mathrm{~L}_{s}$ (bottom). The results are obtained for hydrophobic stripes of width $L=0.46 \mathrm{~L}_{s}$. Left and right panels are for a view perpendicular to the axis of the surface plane and a side view, respectively. Water beads are not shown for clarity. The color code is the same as that used in Figure 2. 

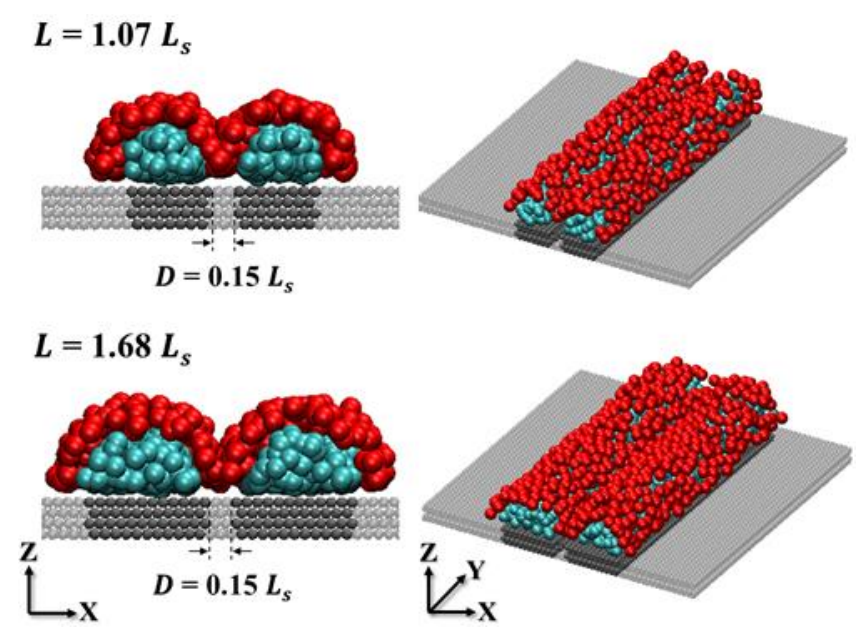

Figure10. Representative simulation snapshots for surfactant aggregates self-assembled on two hydrophobic stripes of width $\mathrm{L}=1.07 \mathrm{~L}_{\mathrm{s}}$ (top) and $1.68 \mathrm{~L}_{\mathrm{s}}$ (bottom) separated by $\mathrm{D}=0.15 \mathrm{~L}_{\mathrm{s}}$. Left and right panels are for a view perpendicular to the axis of the surface plane and a side view, respectively. Water beads are not shown for clarity. The color code is the same as that used in Figure 2. 